\title{
The Araucaria Project. The distance to the Sculptor group galaxy NGC 7793 from near-infrared photometry of Cepheid variables
}

\author{
Bartlomiej Zgirski ${ }^{1, \star}$, Wolfgang Gieren ${ }^{3,4}$, Grzegorz Pietrzyński ${ }^{1,3}$, Paulina Karczmarek ${ }^{2}$, \\ Marek Górski ${ }^{3,4}$, Dariusz Graczyk ${ }^{1,3,4}$, Bogumil Pilecki ${ }^{1}$, Mónica Taormina ${ }^{1}$, Ksenia Suchomska ${ }^{2}$, \\ Piotr Wielgórski ${ }^{1}$, and Piotr Konorski ${ }^{2}$ \\ ${ }^{1}$ Nicolaus Copernicus Astronomical Center of the Polish Academy of Sciences, Warsaw, Poland \\ ${ }^{2}$ Astronomical Observatory of the University of Warsaw, Warsaw, Poland \\ ${ }^{3}$ Universidad de Concepción, Departamento de Astronomia, Concepción, Chile \\ ${ }^{4}$ Millennium Institute of Astrophysics, Santiago, Chile
}

\begin{abstract}
We performed deep near-infrared $J$ and $K$ photometry of a field in the Sculptor Group spiral galaxy NGC 7793 using ESO VLT and HAWK-I instrument. We produced a sample of 14 Cepheids whose locations and periods of pulsation were known from our previous paper on distance determination to this galaxy based on $V$ and $I$ bands. We determined mean $J$ and $K$ magnitudes based on measurements from two nights and produced period-luminosity relations for both filters. Using those near-infrared dependencies together with relations for bands $V$ and $I$ obtained before, we were able to obtain true distance modulus for NGC 7793. We also calculated the mean reddening affecting our Cepheids.
\end{abstract}

We present first near-infrared period-luminosity relations for Cepheids in NGC 7793. Using these data and optical data published before by Pietrzyński et al. (2010, [2]), we measured accurate distance to this galaxy based on four filters $(V, I, J, K)$.

The near-infrared (NIR) data were collected with HAWK-I and ISAAC cameras installed on VLT telescopes in Cerro Paranal Observatory. From the optical catalogue of 14 Cepheids, we were able to identify 10 on our near-infrared images.

All Cepheids have photometry from 2 HAWK-I nights in 2011 and additionally 2 brightest Cepheids have also 1 data point obtained with ISAAC in 2005.

Assuming well defined slopes of near-infrared relations in the Large Magellanic Cloud by Persson et al. (2004, [1]), we fitted straight lines to period-luminosity relations of Cepheids in NGC 7793 using the least-squares method.

We adopted well determined distance to the Large Magellanic Cloud of Pietrzyński et al. (2013, [3]) as a zero point and fiducial period-luminosity relations for near-infrared bands for the Large Magellanic Cloud established by Persson et al. (2004, [1]).

The observed distance modulus can be expressed in the following way:

$$
(m-M)_{\lambda}=(m-M)_{0}+R_{\lambda} E(B-V),
$$

^bzgirski@camk.edu.pl 

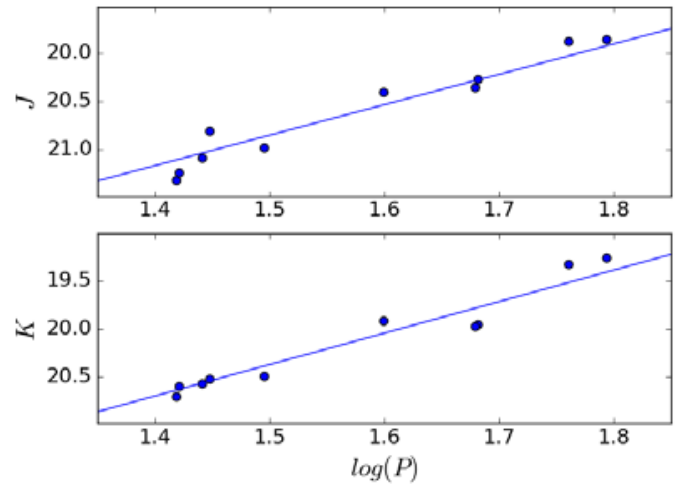

Figure 1. Near-infrared periodluminosity relations for Cepheids in NGC 7793. Corresponding slopes were adopted from Persson et al. (2004, [1]). Periods are in days.

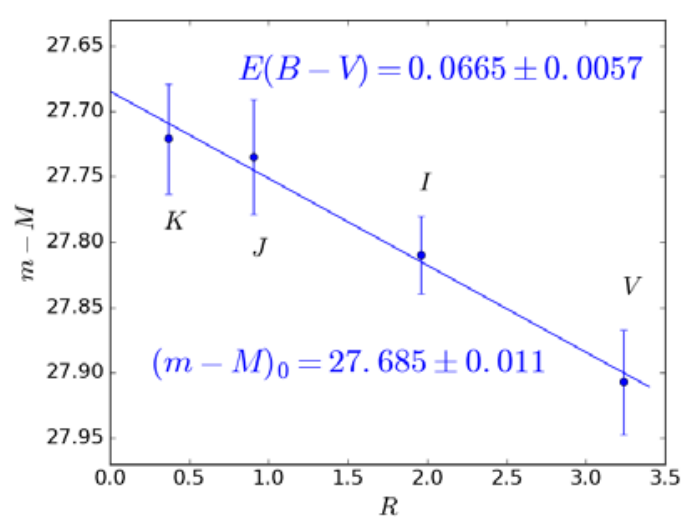

Figure 2. Apparent distance moduli to NGC 7793 as derived in the VIJK photometric bands, plotted against the ratio of total to selective extinction as adopted from Schlegel et al. (1998, [4]). The intersection and slope of the best-fitting line give the true distance modulus and the average total reddening, respectively.

where $(m-M)_{0}$ is the true distance modulus, $E(B-V)$ is color excess and $R_{\lambda}$ is a ratio of extinction in given band to the color excess.

Assuming the reddening law of Schlegel et al. (1998, [4]), we calculate the color excess,

$$
E(B-V)=0.0665 \pm 0.0057
$$

and true distance modulus to NGC 7793,

$$
(m-M)_{0}=27.685 \pm 0.011 \pm 0.070,
$$

which is presently the most accurate distance measurement to NGC 7793.

Acknowledgments: The research leading to these results has received funding from the European Research Council (ERC) under the European Union's Horizon 2020 research and innovation programme (grant agreement No 695099) . Research supported from the IdP II 2015000264 grant of the Polish Ministry of Science and Higher Education.

\section{References}

[1] Persson, S. E., Madore, B. F., Krzeminski, W., et al., AJ, 128, 2239 (2004)

[2] Pietrzyński, G., Gieren, W., Hamuy, M., et al., AJ, 140, 1475 (2010)

[3] Pietrzyński, G., Graczyk, D., Gieren, W., et al., Nature, 495, 76 (2013)

[4] Schlegel, D. J., Finkbeiner, D. P., \& Davis, M., ApJ, 500, 525 (1998) 